\title{
Fusogenic Viral Protein-based Near-infrared Active Nanocarriers for Biomedical Imaging
}

Suman Bishnoi ${ }^{1}$, Anshu Kumari ${ }^{1,4}$, Sheeba Rehman ${ }^{1}$, Aliva P. Minz ${ }^{3}$, Shantibhusan Senapati ${ }^{3}$, Debasis Nayak ${ }^{1 *}$, Sharad Gupta ${ }^{1,2 *}$

${ }^{1}$ Department of Biosciences and Biomedical Engineering, Indian Institute of Technology Indore, Indore 453 552, India

${ }^{2}$ School of Biotechnology, Jawaharlal Nehru University, New Delhi 110067, India

${ }^{3}$ Institute of Life Sciences, Nalco Square, Bhubaneswar, Odisha 751023, India

${ }^{4}$ School of Medicine, University of Maryland Baltimore, Maryland 21201, USA

*Corresponding authors

Dr. Sharad Gupta

E-mail ID: shgupta@iiti.ac.in

Dr. Debasis Nayak

E-mail ID: nayakdn@iiti.ac.in

Supporting Information consists of three pages and includes two figures.

\section{List of Figures:}

Figure S1. SDS-PAGE analysis of purified VSV-G VNPs.

Figure S2. Calibration curve of indocyanine green (ICG) dye in water. 
1. Sodium dodecyl sulfate (SDS)-polyacrylamide gel electrophoresis: The purity of the VSV-G VNPS was analyzed by SDS-PAGE. On $10 \%$ SDS gel, the VNPs purified from conditioned media of VSV-G transfected HEK293T cells and wild-type VSV was loaded. The protein bands were visualized with Coomassie blue staining. The result shows an overwhelmingly higher amount of purified VSV-G protein with a molecular weight of 67 $\mathrm{kDa}$ and a trace amount of membrane proteins as VNPs acquire envelope from the mammalian host cells (Lane 2, Figure S1). The purified wild-type VSV constitutes a VSV-G protein of the $67 \mathrm{kDa}$ size similar to VNPs and other structural and non-structural proteins of virus (Lane 3, Figure S1).

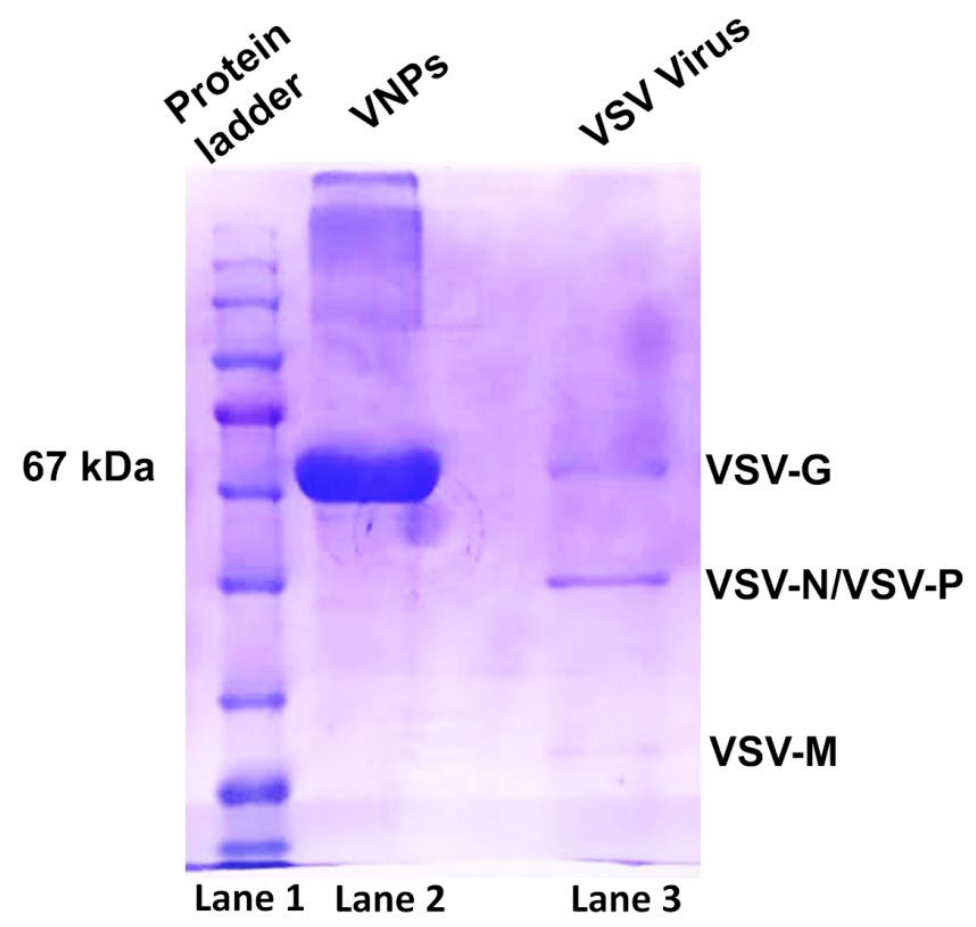

Figure S1. SDS-PAGE analysis of purified VSV-G VNPs. Protein ladder (lane 1), purified VNPs depicting the overwhelmingly higher amount of VSV-G protein with a molecular weight of 67 $\mathrm{kDa}$ and residual membrane proteins (lane 2), purified wild-type VSV virus displaying VSV-G (67 kDa), VSV-N/VSV-P protein, and VSV-M protein (lane 3).

2. Spectroscopic analysis: The absorption spectrum of free ICG in water was recorded using a UV-Vis-NIR spectrophotometer. The calibration curve of absorbance versus different concentrations of free ICG in water was plotted using Origin 8.0 software. The calibration curve was used to estimate the encapsulation efficiency of ICG within NAVN. 


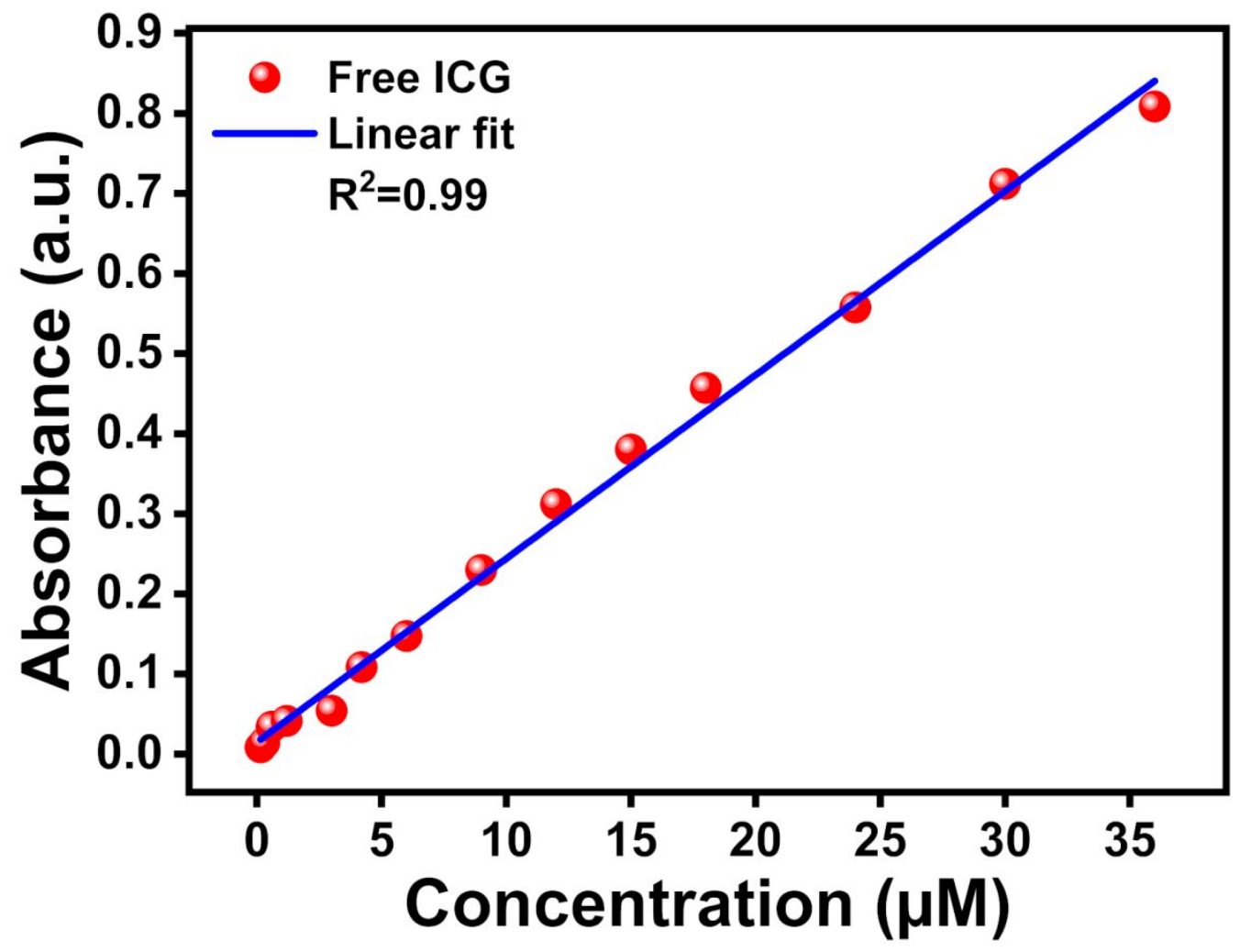

Figure S2. Calibration curve of indocyanine green (ICG) dye in water. 